\title{
Performances Analysis of High-Speed 1-bit Full Adder
}

\section{Kratikatiyar and Praveen Kumar}

\begin{abstract}
Analysis of a hybrid single bit full adder is discussed here. The adders are implemented and analysis to reduce the power dissipation and output voltage degradation. The simulation result of this single bit full adder using 16 Transistor was performed considering different technology with special stress on the design methodology and analysis. The Hybrid logic designs the characteristics of altered logic or design styles, enhance overall performance. The delay introduced due to propagation is further minimized by transistor size also discussed in this paper. We also discussed about their various performance parameter such as delay, power etc.

Keywords

Delay approximation;

Hybrid full-adder;

Low-power full-adder;

Multiplexer based full-adder design;

Power Delay Product.
\end{abstract}

\section{INTRODUCTION}

Adder is considered as one of the basic units required in the basic VLSI circuit [1]. It mainly has three input ports and two output port. That outputs are sum and carry-out which are produced simultaneously [2]. The first step of addition is adding two binary digits. The carry is generated only when the two bits are high [3]. A half adder is based on two binary inputs and gives two binary output sum and carry[4]. Boolean expression of sum and carry can be written as

$$
\begin{gathered}
\mathrm{S}=\mathrm{A} \oplus \mathrm{B} \oplus \mathrm{C} \\
\mathrm{C}=\mathrm{AB}+\mathrm{BC}+\mathrm{AC}
\end{gathered}
$$

The response speed of adder circuit is responsible on the carry signal. The generated carry signal suffer formed propagation delay which is typically decrease by overall path length reduction [5]. There mainly two logic styles are used to design full adder, by using linear threshold gate or by using CMOS [6]. The design based on both CMOS logic and transmission gate logic and Carbon Nano-tube field effect transistor (CNTFET) [7]. Performance parameter such as delay, power and products power and delay are calculated.

\section{DESIGN APPROACH}

Adder circuit have three blocks. The block diagram of the tested CMOS based full adder is shown in Fig 1. Where both the module $1 \& 2$ is the Exclusive-NOR (XNOR) module which are used to produce the resultant sum signal (SUM) and other module i.e. module 3 is used to produce the output carry signal [8]. The main objectives of three individual modules area each module is design in such a way that it produces optimum performance parameter [9]. These optimized models are discussed here.

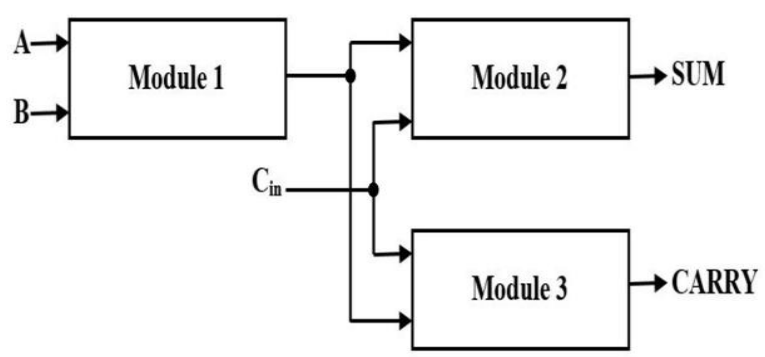

Fig 1. Block Diagram of full adder.

\section{A. Modified XNOR Module}

XNOR module presented in this full adder circuit is accountable due to the major of the power consuming unit of the complete device. It design such a way that it could avoid the voltage degradation. XNOR circuit power utilization adequately by the circuit is minimized by using inverter. Different XOR/XNOR design methodology reported in [10][13].

The detail circuit of the full adder circuit is illustrated in Fig 2. By considering the single bit full adder's truth table carry generated as B when, A and B has same value otherwise output carry is same as input carry. The output carry signal is produced by Q9-Q11. The overall time taken for carry propagation path reduces considerably.

The sum output in this circuit is realize by Exclusive NOR or XNOR modules.

The simulation is carried out in different technology with importance on the hybrid design methodology. To optimize one of the important parameter power-delay products (PDP), which is also, imply energy consumptions minimization. The Hybrid logic design the characteristic of altered logic is enhance overall output performance. 
The XNOR module (module $1 \& 2$ ) are the power consumption mainly reduced properly tries to achieve full swing of the desire voltage of output signals. The input carry signal (Cin) pass through a particular transistor for reducing the propagation path. The delay generally predominantly affects the overall performance of adder circuit.

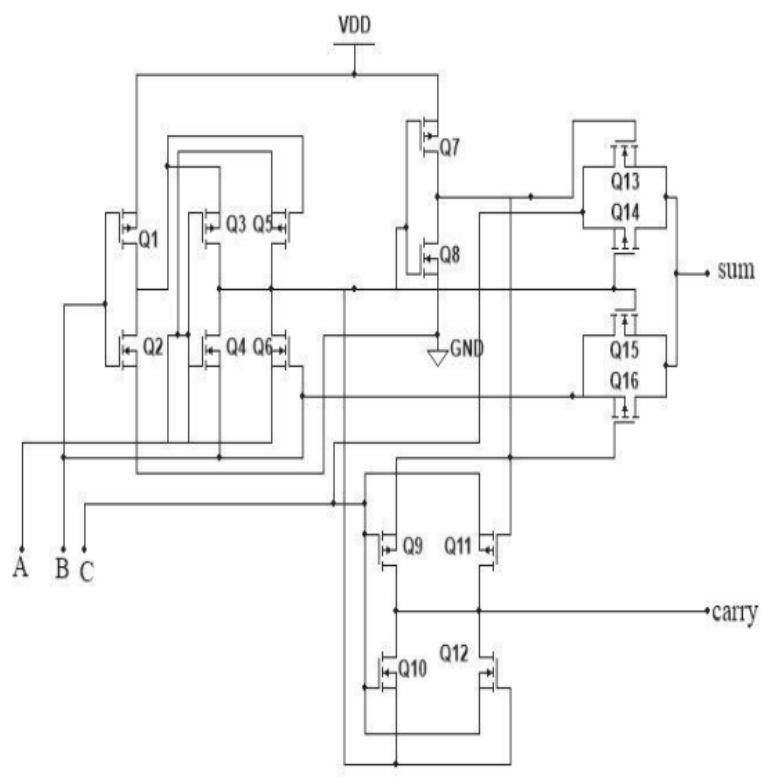

Fig 2. Circuit diagram using 16 transistor full adder.

\section{A. Average Power and Propagation Delay}

The delay of the hybrid full adder is less in compare with CMOS hybrid full adder. The average power full adder at $45 \mathrm{~nm}$ technology is $0.778 \mathrm{w}$ and $0.073 \mathrm{w}$ respectively. The delay time estimated here at $45 \mathrm{~nm}$ technology is $40.1 \mathrm{ps}$ and $16.83 \mathrm{ps}$. Advantage of this circuit less transistor count and small area and disadvantage of this full-adder experience driving capabilities in fan-out situation and their performances degrade intensely when they are cascaded. Output of the adder is shown in Fig 3. The simulation results prove that the one-bit hybrid full adder works efficiently.

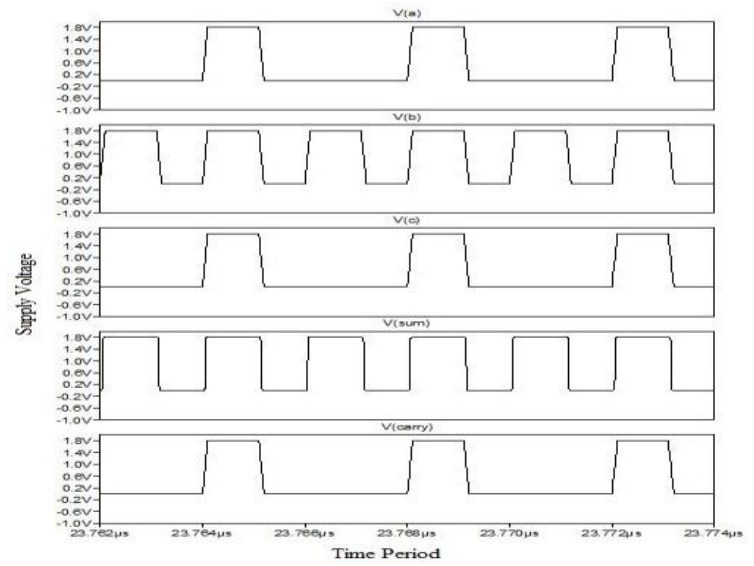

Fig 3. Simulation result of 16 transistor full adder.
In the cascaded operations in carry propagation mode, the operation speed performance of the circuit degraded with increase in stages. The cascaded full adders operating in carry propagation mode. Considering the source, Vin is step signal, the delay $\tau \operatorname{pd}(\mathrm{m})$ time constant, $\tau$ considering as first order circuit and applying the delay approximation using Elmore formulae [3]:

$$
\begin{aligned}
& \tau_{p d}(m)_{\text {total }}=\tau_{p d}(m)+\tau_{p d b u f} \\
& =0.69 m R\left[\frac{\left(C_{1}+C_{2}\right)(m+1)}{2}+\left(C_{\text {inbuf }}-C_{1}\right)\right]+\tau_{p d b u f}
\end{aligned}
$$

It was found extremely low average power consumption very low and negligible delay (224 ps) in $180-\mathrm{nm}$ technology for $1.8 \mathrm{~V}$. it resulting power consumption of 1.7664 and delay of 91.3 ps In 90-nm technology on $1.2 \mathrm{~V}$. When this above explained design extended for 32 bit full adder it is found that it working efficiently on $180 \mathrm{~nm}$ technology for $1.8 \mathrm{~V}$ $(1.2 \mathrm{~V})$ supply voltage and producing Delay of only $5.578 \mathrm{~ns}(2.45 \mathrm{~ns})$ and power consumption of $112.79 \mu \mathrm{W}(53.36 \mu \mathrm{W})$. The implemented design shows significant improvement on various parameters such as power and speed compare to the existing full adder designs.

Table 1. Simulation results

\begin{tabular}{|c|c|c|c|}
\hline Methods & Delay (ps) & $\begin{array}{c}\text { Average } \\
\text { power } \\
(\mu W)\end{array}$ & PDP (fJ) \\
\hline $\begin{array}{c}\text { CMOS-logic } \\
\text { based Full } \\
\text { Adder }\end{array}$ & 16.7 & 0.076 & 0.00122 \\
\hline $\begin{array}{c}\text { CNTFET } \\
\text { based Full } \\
\text { adder }\end{array}$ & 224 & 4.1563 & 0.931 \\
\hline
\end{tabular}

\section{Conclusion}

Overall operational speed and output performance of the system are dependent on the propagation delay and it usually reduced by minimizing the path length. The delay is also further minimized by transistor size minimization. The estimated Power Delay Product improvement is possible was $25 \%$ when the same design was verified using $90 \mathrm{~nm}$ technology. The presented design verified using $32 \mathrm{~nm}$ technology.

\section{REFERENCES}

[1] Bayoumi, M., Jullien, G., \& Miller, W. (1987). A VLSI implementation of residue adders. IEEE Transactions on Circuits and Systems, 34(3), 284-288. 
[2] Montoye, R. K., \& Cocke, J. (1990). U.S. Patent No. 4,969,118. Washington, DC: U.S. Patent and Trademark Office.

[3] Bhattacharyya, P., Kundu, B., Ghosh, S., Kumar, V., \& Dandapat, A. (2015). Performance analysis of a low-power high-speed hybrid 1-bit full adder circuit. IEEE Transactions on very large scale integration (VLSI) systems, 23(10), 20012008.

[4] Goel, S., Gollamudi, S., Kumar, A., \& Bayoumi, M. (2004, July). On the design of low-energy hybrid CMOS 1-bit full adder cells. In Circuits and Systems, 2004. MWSCAS'04. The 2004 47th Midwest Symposium on (Vol. 2, pp. II-II). IEEE.

[5] Chang, C. H., Gu, J., \& Zhang, M. (2005). A review of $0.18-/ \mathrm{spl} \mathrm{mu} / \mathrm{m}$ full adder performances for tree structured arithmetic circuits. IEEE Transactions on very large scale integration (VLSI) systems, 13(6), 686-695.

[6] Zimmermann, R., \& Fichtner, W. (1997). Lowpower logic styles: CMOS versus pass-transistor logic. IEEE journal of solid-state circuits, 32(7), 1079-1090.

[7] Al-Rabadi, A. N. (2007). Carbon nano tube (CNT) multiplexers for multiple-valued computing. Facta universitatis-series: Electronics and Energetics, 20(2), 175-186.

[8] Wang, J. M., Fang, S. C., \& Feng, W. S. (1994). New efficient designs for XOR and XNOR functions on the transistor level. IEEE Journal of solid-state Circuits, 29(7), 780-786.

[9] Prashanth, P., \& Swamy, P. (2011, December). Architecture of adders based on speed, area and power dissipation. In Information and Communication Technologies (WICT), 2011 World Congress on (pp. 240-244). IEEE.

[10] Zhao, W., Chappert, C., Javerliac, V., \& Noziere, J. P. (2009). High speed, high stability and low power sensing amplifier for MTJ/CMOS hybrid logic circuits. IEEE Transactions on Magnetics, 45(10), 3784-3787.

[11] Goel, S., Kumar, A., \& Bayoumi, M. A. (2006). Design of robust, energy-efficient full adders for deep-submicrometer design using hybrid-CMOS logic style. IEEE Transactions on Very Large Scale Integration (VLSI) Systems, 14(12), 13091321.

[12] Mewada, M., \& Zaveri, M. (2015). An Improved Input Test Pattern for Characterization of Full Adder Circuits. International Journal of Research and Scientific Innovation-IJRSI, 3(1), 222-226.
[13] Wyatt Jr, J. L. (1987). Signal propagation delay in RC models for interconnect. Circuit Analysis, Simulation and Design, 3, 254-291.

\author{
Kratikatiyar \\ Trinity College of Engineering, Bhopal, India \\ kratikatiyar@gmail.com \\ Praveen Kumar \\ Trinity College of Engineering, Bhopal, India \\ praveenkumar8398@gmail.com
}

